\title{
Unmasking the history of 3C 293 with LOFAR sub-arcsecond imaging
}

\author{
Pranav Kukreti ${ }^{1,2} \odot$ ｜ Raffaella Morganti ${ }^{1,2} \odot ｜$ Timothy W. Shimwell ${ }^{2,3}$ | \\ Leah K. Morabito ${ }^{4,5}$ | Robert J. Beswick ${ }^{6}$ | Marisa Brienza ${ }^{7,8}$ \\ Martin J. Hardcastle ${ }^{9}$ | Frits Sweijen ${ }^{3}$ | Neal Jackson ${ }^{6}$ | George K. Miley ${ }^{3}$ | \\ Javier Moldon $^{10}$ | Tom Oosterloo ${ }^{1,2}$ | Francesco de Gasperin ${ }^{8,11}$
}

\begin{abstract}
${ }^{1}$ Kapteyn Astronomical Institute, Faculty of Science and Engineering, University of Groningen, Groningen, The Netherlands

${ }^{2}$ ASTRON, the Netherlands Institute for

Radio Astronomy, NWO, Dwingeloo, The Netherlands

${ }^{3}$ Leiden Observatory, Faculty of Science,

Leiden University, Leiden, The

Netherlands

${ }^{4}$ Department of Physics, Centre for

Extragalactic Astronomy, Durham

University, Durham, UK

${ }^{5}$ Department of Physics, Institute for

Computational Cosmology, University of

Durham, Durham, UK

${ }^{6}$ Jodrell Bank Centre for Astrophysics,

School of Physics and Astronomy,

University of Manchester, Manchester, UK

${ }^{7}$ Dipartimento di Fisica e Astronomia,

Università di Bologna, Bologna, Italy

${ }^{8} \mathrm{INAF}$ - Istituto di Radioastronomia,

Bologna, Italy

${ }^{9}$ Centre for Astrophysics Research,

University of Hertfordshire, Hatfield, UK

${ }^{10}$ Instituto de Astrofísica de Andalucía

(IAA, CSIC), Glorieta de las Astronomía,

Granada, Spain

${ }^{11}$ Hamburger Sternwarte, Faculty of

Mathematics, Computer Science, and

Abstract

Active galactic nuclei show episodic activity, which can be evident in galaxies that exhibit restarted radio jets. These restarted jets can interact with their environment, leaving signatures in the radio spectral energy distribution. Tracing these signatures requires resolved spectral index measurements over a broad frequency range. We present such a study for the radio galaxy 3C 293. Using the International Low Frequency Array Telescope (ILT), we probe spatial scales as fine as $\sim 0.2^{\prime \prime}$ at $144 \mathrm{MHz}$ and to constrain the spectrum, we combine these data with Multi-Element Radio Linked Interferometer Network and Very Large Array archival data at frequencies up to $8.4 \mathrm{GHz}$. In the inner lobes $(\sim 2 \mathrm{kpc})$, we detect the presence of a compact steep spectrum (CSS) source with a spectral turnover at $\sim 225 \mathrm{MHz}$, caused by free-free absorption from the rich surrounding medium. We find evidence of interaction of the lobes with the rich interstellar medium of the host galaxy. The outer lobes (extending up to $\sim 100 \mathrm{kpc}$ ) have a remarkably uniform spatial distribution of spectral index and only mild spectral curvature (of $\sim 0.2$ ). Overall, it appears that $3 \mathrm{C} 293$ has gone through multiple (two to three) epochs of activity. This study adds 3C 293 to the new sub-group of restarted galaxies with short interruption time periods ( $\lesssim 1 \mathrm{Myr}$ ). This is the first time a spatially resolved study has been performed that simultaneously studies a young CSS source as well as the older outer lobes at such low frequencies.
\end{abstract}

Natural Sciences, Universität Hamburg,

Hamburg, Germany

\section{Correspondence}

Pranav Kukreti, Kapteyn Astronomical

Institute, University of Groningen,

Groningen, The Netherlands and

ASTRON, the Netherlands Institute for

Radio Astronomy, Dwingeloo, The

Netherlands.

Email: kukreti@astro.rug.nl

\section{K E Y W O R D S}

galaxies: active, galaxies: individual: 3C 293-techniques: high angular resolution, radio continuum: galaxies

This is an open access article under the terms of the Creative Commons Attribution License, which permits use, distribution and reproduction in any medium, provided the original work is properly cited.

(C) 2021 The Authors. Astronomische Nachrichten published by Wiley-VCH GmbH. 


\section{1 | INTRODUCTION}

Active galactic nuclei (AGN) have been demonstrated to show episodic activity and have been identified to have different phases of their life cycle. Lobes of remnant plasma from a previous phase of activity coexisting with a newly born pair of radio jets are typical indicators of restarted or episodic activity in such galaxies. Over the past few years, a new sub-group of candidate restarted radio galaxies have been found, which do not show spatially resolved spectral properties expected from old remnant plasma, such as an ultra steep spectrum ${ }^{1}(\alpha>1.2)$ and a steep curvature in the radio spectra $(\Delta \alpha \geq 0.5)$. Instead, these sources show bright inner jets and very diffuse outer lobes with a homogeneous spatial distribution of spectral index, such as Centaurus A (McKinley et al. 2018), B2 0258+35 (Brienza et al. 2018), and more recently NGC 3998 (Sridhar et al. 2020). Although the physical mechanism responsible for these properties is still under debate, some studies indicate that the older outer lobes could still be fueled at low levels by the active inner jets (McKinley et al. 2018). This is different from the model of typical restarted galaxies where fueling of the older lobes has stopped. Therefore, this sub-group poses a new challenge to our understanding of the life-cycle of radio galaxies. We study one such candidate restarted galaxy $-3 \mathrm{C} 293$, located at a redshift of $z=0.045$.

\section{2 | DATA}

In order to trace the radio emission and characterize the spectral properties over a broad range of frequencies and on large and small scales, we used data from different telescopes. We used new observations with the International Low Frequency Array Telescope (ILT) High Band Antenna (HBA) at 120-168 MHz. We combine these with archival Very Large Array (VLA) data at $1.36 \mathrm{GHz}$ and $4.85 \mathrm{GHz}(\mathrm{C}+\mathrm{D}$ array) for a low-resolution spectral study. For the high-resolution complementary data, we use archival Multi-Element Radio Linked Interferometer Network (MERLIN) $1.36 \mathrm{GHz}$ data, and VLA $4.85 \mathrm{GHz}$ (A array) and $8.45 \mathrm{GHz}$ data.

\section{1 | LOFAR HBA observations}

We performed targeted observations of 3C 293 with the ILT HBA for a total of $8 \mathrm{hr}$ on July 30, 2020 and August 2, 2020 (Project code-LC14_015). The 60 Dutch stations and 12 international stations were used as a part of the ILT array.

${ }^{1}$ Throughout the paper we use the notation $S \propto v^{-\alpha}$.

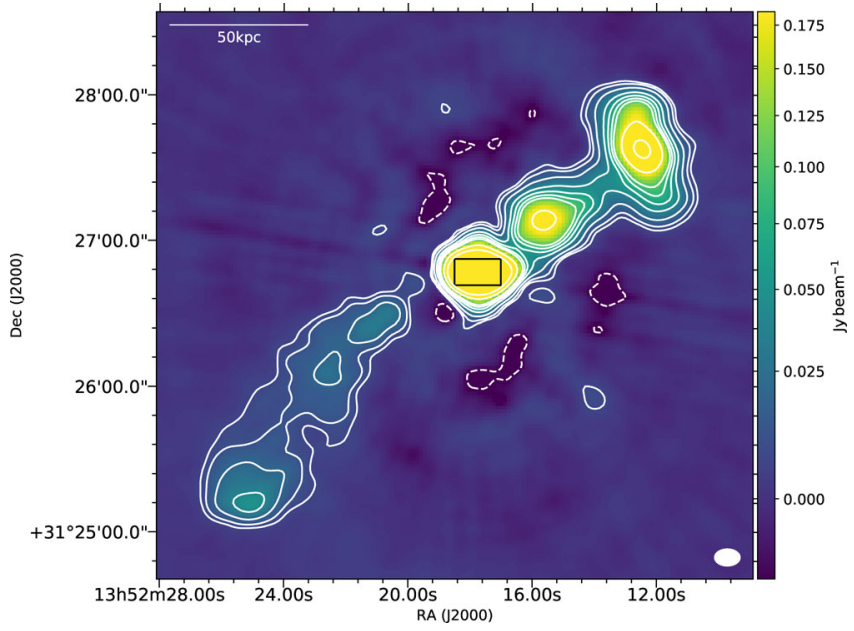

F I G U R E 1 LOFAR Dutch array image from LoTSS-DR2 data showing the large-scale $\left(\sim 250^{\prime \prime}\right)$ structure of 3C 293 at $144 \mathrm{MHz}$ with a resolution of $10.5^{\prime \prime} \times 7^{\prime \prime}$. The contours levels are: $(-3,3,5,10$, $20,30,40,50,100,200) \times \sigma_{\mathrm{RMS}}$ where $\sigma_{\mathrm{RMS}}=2 \mathrm{mJy}$ beam $^{-1}$ is the root-mean-square (RMS) noise in the image. The black rectangle in the center marks the region that is shown in the high-resolution image. LoTSS, LOFAR two-meter sky survey

The data were averaged and calibrated using the LOFAR long baseline pipeline ${ }^{2}$ (Morabito et al. 2021), which performs in-field delay calibration using a bright calibrator in the field of view and cross-matching it with the LOFAR Long-Baseline Calibrator Survey (Jackson et al. 2016; Jackson et al. 2021) and LOFAR Two-meter Sky Survey (LoTSS; Shimwell et al. 2017). The low-resolution Dutch array and high-resolution International array images of 3C 293 are shown in Figures 1 and 2, respectively.

\section{3 | SPECTRAL PROPERTIES}

\section{1 $\quad$ Central region}

We constructed a two-point spectral index map of the central region using high-resolution images at 144 and $1400 \mathrm{MHz}$, shown in Figure 3. We can clearly see two distinct spectral index populations-the inner lobes with an index of $\alpha_{1360}^{144} \lesssim 0.3$, and the diffuse emission on either side with a spectral index of $\alpha_{1360}^{144} \approx 0.6-0.8$. The spectra for the inner lobes are below the theoretical limit for injection index $\left(\alpha_{\mathrm{inj}}=0.5\right)$. This suggests that the spectra peak in between 144 and $1360 \mathrm{MHz}$, which implies the presence of low-frequency absorption. An absorbed spectrum is seen in compact steep spectrum (CSS) or gigahertz peaked spectrum (GPS) radio galaxies. This peak

\footnotetext{
${ }^{2}$ https://github.com/lmorabit/lofar-vlbi .
} 


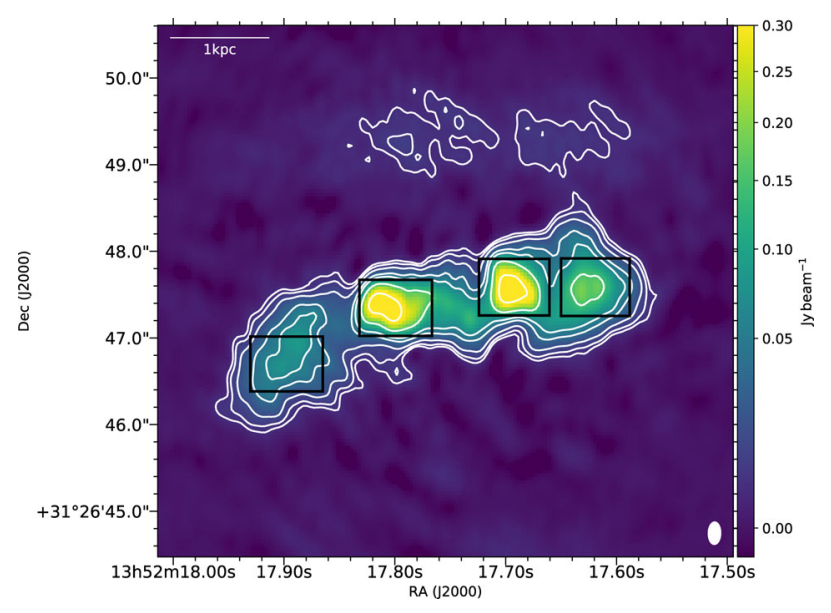

F I G U R E 2 LOFAR international stations image at $144 \mathrm{MHz}$ of the central $4.5 \mathrm{kpc}$ region with a resolution of $0.26^{\prime \prime} \times 0.15^{\prime \prime}$. The small-scale inner lobes and diffuse emission are visible and contour levels are: $(-3,3,5,10,25,40,100,250) \times \sigma_{\mathrm{RMS}}$ where $\sigma_{\mathrm{RMS}}=1.5$ mJy beam ${ }^{-1}$ is the RMS noise. The black rectangles mark the regions used to extract flux densities for the model fits

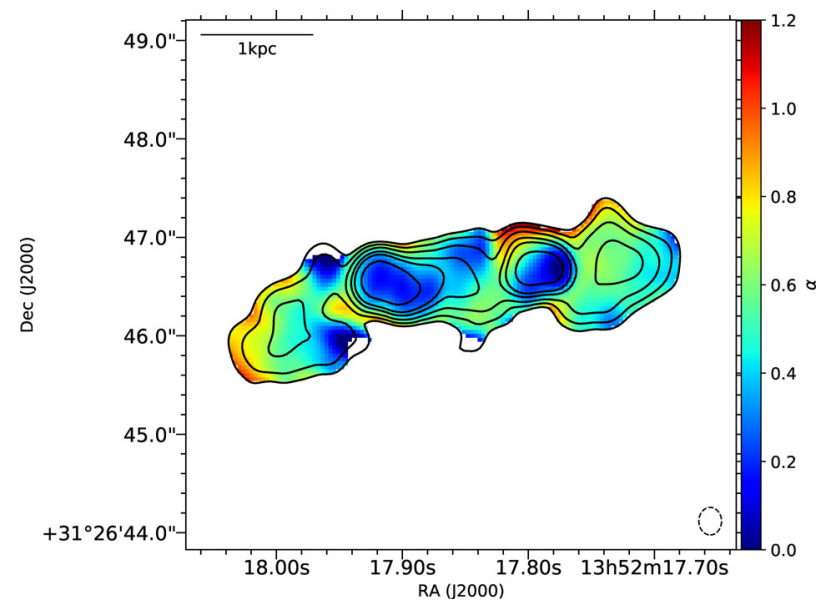

F I G U R E 3 Spectral index map of the high-resolution central region of 3C 293 from 144 to $1360 \mathrm{MHz}$ at $0.28^{\prime \prime} \times 0.23^{\prime \prime}$ resolution. The contour levels are: $(5,10,20,40,65,150) \times \sigma_{\mathrm{RMS}}$ where $\sigma_{\mathrm{RMS}}$ $=1.4 \mathrm{mJy}$ beam $^{-1}$ is the local RMS noise in the $1360 \mathrm{MHz}$ image

is attributed to the absorption of synchrotron radiation in the source, which is broadly a manifestation of either synchrotron self-absorption (SSA) or free-free absorption (FFA) (Kellermann 1966).

\section{2 | Outer lobes}

We find a lack of spatial gradient in the spectral index throughout the outer lobe, shown in Figure 1, from 144 to $4850 \mathrm{MHz}$. The spectral index appears to have a homogeneous distribution, at our resolution, which corresponds to a physical scale of $\sim 10 \mathrm{kpc}$.

\section{4 | DISCUSSION}

\section{1 | Interplay of radio plasma and gas in the central region}

We have extracted flux densities from regions of $0.8^{\prime \prime} \times 0.6^{\prime \prime}$ across the inner lobes, shown in Figure 2, and fitted SSA and FFA absorption models to the spectra. Both models fit the data reasonably well, and since we do not have the spectral index information below the turnover, we have used physical quantities estimated using the model fit parameters to discriminate between these models.

Using SSA fit parameters we estimate the magnetic field strength of the source (Callingham et al. 2015; Kellermann \& Pauliny-Toth 1981). We find a magnetic field of 4.3-4.9 $\times 10^{2} \mathrm{G}$ for the inner lobes, which are unrealistically high for CSS/GPS sources and also significantly higher than our equipartition estimates, by $\sim 10^{7}$. Hence, it is unlikely that SSA is the dominant absorption mechanism in the inner lobes.

For FFA, we find that the optical depth is systematically higher for the western inner lobe than the eastern inner lobe, with a $3 \sigma$ significance (see vertical lines in Figure 4). Over the years, resolved absorption studies of CSS/GPS sources have found that a difference between the optical depths of the lobes is due to the larger path length in the ionized medium along the line of sight for the emission from the receding lobe, such as OQ 208 (Kameno et al. 2000), 3C 84 (Vermeulen et al. 1994; Walker et al. 1999), and NGC 4261 (Jones et al. 2000). The asymmetry in the derived optical depths is consistent with the orientation of the inner jets (Beswick et al. 2004). Using FFA fit parameters, we estimate the emission measure and a path length of $\approx 50 \mathrm{pc}$ for eastern and $\approx 80 \mathrm{pc}$ for western inner lobe. (Emonts et al. 2005) have found evidence for narrow line region clouds out to a few kpc in the central region of 3C 293, which is in agreement with our estimated path lengths. We conclude that FFA is the dominant absorption mechanism in the inner lobes of 3C 293.

Multiple studies of CSS and GPS sources have found a correlation between the linear size of the source and the peak frequency of the spectrum (Bicknell et al. 1997; O’Dea $\&$ Baum 1997). Using this correlation, we find a minimum peak frequency of $349_{-24}^{+26} \mathrm{MHz}$, corresponding to the $\sim 2.5$ kpc size of the inner lobes.

\subsection{Are the outer lobes still alive?}

The lack of a spatial gradient in spectral index is contrary to the trend expected, as plasma in different regions would have different ages and would be expected to show different curvatures in their spectra. This suggests that the plasma in the lobe is highly turbulent, which reaccelerates 


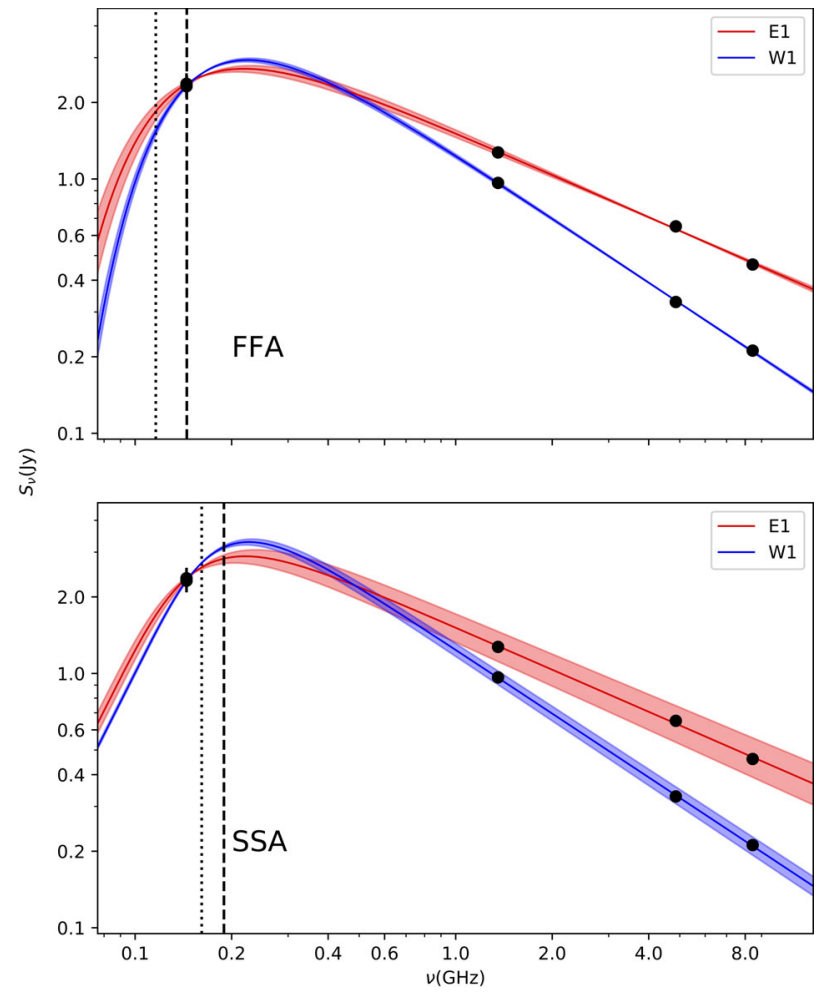

F I G U R E 4 Best fits of free-free absorption (top panel) and synchrotron self-absorption (bottom panel) absorption models to the eastern (E1) and western (W1) inner lobes of 3C 293. The vertical lines show the $v_{p}$ frequency (a measure of the optical depth), for eastern (dotted) and western (dashed) lobe

old electrons (due to shock acceleration) and mixes different electron populations. The diffuse morphology of the north-western lobe also suggests that the flow is not very well collimated and more turbulent. Overall, the spectral properties of the outer lobes are a result of one or maybe both of the two scenarios-multiple episodes of jet activity or turbulent jet flow due to disruption by strong jet-ISM interaction. Any interruption of jet activity has happened only a few Myr ago, and the lobe emission does not show properties of remnant plasma. Shock-powered turbulence (Lanz et al. 2015) may have kept the spectra from steepening and the spectral distribution uniform spatially. Overall, also considering the young CSS source in the center, we conclude that $3 \mathrm{C} 293$ has had two to three epochs of activity.

\section{SUMMARY}

Our analysis has revealed that 3C 293 is not a typical restarted galaxy with a new phase of activity embedded in lobes of diffuse emission and properties of remnant plasma. We have observed for the first time, absorption in the inner lobes of 3C 293 with a peak frequency of $\sim 230-240 \mathrm{MHz}$. FFA from the narrow line region in the rich surrounding medium of the host galaxy is likely the dominant absorption mechanism. From the age, size, and presence of a turnover in the spectrum of the inner lobes of 3C 293, we conclude that it is a young CSS source whose growth is affected by the dense surrounding medium. Although 3C 293 has outer lobes that show spectral properties of active fueling (and are hence alive), the presence of a young CSS source in the center has confirmed that it is indeed a restarted galaxy. Finding similar sources and correctly categorizing them separately from typical restarted galaxies is important, since shorter interruption time periods would affect the overall understanding of AGN life cycle timescales, which is a crucial input for AGN feedback models. This will be possible with the International stations of ILT providing sub-arcsecond resolution at $144 \mathrm{MHz}$ (HBA), and in the future at $57 \mathrm{MHz}$ (LBA).

\section{ORCID}

Pranav Kukreti (1) https://orcid.org/0000-0001-6020-4417 Raffaella Morganti (1) https://orcid.org/0000-0002-94826844

\section{REFERENCES}

Beswick, R. J., Peck, A. B., Taylor, G. B., \& Giovannini, G. 2004, MNRAS, 352(1), 49.

Bicknell, G. V., Dopita, M. A., \& O’Dea, C. P. O. 1997, Ap J, 485(1), 112.

Brienza, M., Morganti, R., Murgia, M., et al. 2018, $A \& A$, 618, A45.

Callingham, J. R., Gaensler, B. M., Ekers, R. D., et al. 2015, ApJ, 809(2), 168.

Emonts, B. H. C., Morganti, R., Tadhunter, C. N., Oosterloo, T. A., Holt, J., \& van der Hulst, J. M. 2005, MNRAS, 362(3), 931.

Jackson, N., Badole, S., Morgan, J., et al. 2021, $A \& A$ in press.

Jackson, N., Tagore, A., Deller, A., et al. 2016, $A \& A$, 595, A86.

Jones, D. L., Wehrle, A. E., Meier, D. L., \& Piner, B. G. 2000, Ap J, 534(1), 165.

Kameno, S., Horiuchi, S., Shen, Z.-Q., Inoue, M., Kobayashi, H., Hirabayashi, H., \& Murata, Y. 2000, PASJ, 52, 209.

Kellermann, K. I. 1966, AuJPh, 19, 195.

Kellermann, K. I., \& Pauliny-Toth, I. I. K. 1981, ARAA, 19(1), 373.

Lanz, L., Ogle, P. M., Evans, D., Appleton, P. N., Guillard, P., \& Emonts, B. 2015, ApJ, 801(1), 17.

McKinley, B., Tingay, S. J., Carretti, E., et al. 2018, MNRAS, 474(3), 4056.

Morabito, L. K., Jackson, N., Mooney, S., et al. 2021, $A \& A$ in press.

O’Dea, C. P., \& Baum, S. A. 1997, Astron. J., 113, 148.

Shimwell, T. W., Röttgering, H. J., Best, P. N., et al. 2017, $A \& A$, 598, A104.

Sridhar, S. S., Morganti, R., Nyland, K., Frank, B. S., Harwood, J., \& Oosterloo, T. 2020, A\&A, 634, A108.

Vermeulen, R. C., Readhead, A. C. S., Backer, D. C., Vermeulen, R. C., Readhead, A. C. S., \& Backer, D. C. 1994, Evidence for a Counter-Jet in 3 C84 (Tech. Rep.). Compact Extragalactic Radio Sources, Proceedings of the NRAO workshop (Socorro, New Mexico).

Walker, R. C., Dhawan, V., Romney, J. D., Kellermann, K. I., \& Vermeulen, R. C. 1999, Ap J, 530(1), 233. 


\section{AUTHOR BIOGRAPHY}

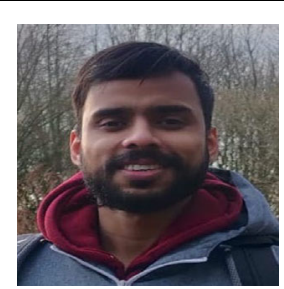

Pranav Kukreti is a $\mathrm{PhD}$ student at the Kapteyn Astronomical Institute. The main goal of his $\mathrm{PhD}$ is to study the gas and life cycle of radio galaxies, especially galaxies that show multiple phases of activity and connect the evolutionary sequence of radio galaxies with information about the surrounding gas.
How to cite this article: Kukreti, P., Morganti, R., Shimwell, T. W., et al. 2021, Astron. Nachr., 1. https://doi.org/10.1002/asna.20210107 